\title{
Atomic-scale Observation of Polymer-chain Conformations Using Scanning Transmission Electron Microscopy
}

Tomohiro Miyata $^{1}$, Raita Goseki ${ }^{2}$, Takashi Ishizone ${ }^{2}$ and Hiroshi Jinnai ${ }^{1}$

${ }^{1}$ Tohoku University, Sendai, Miyagi, Japan, ${ }^{2}$ Tokyo Institute of Technology, Meguro, Tokyo, Japan

Polymeric materials are widely used from clothing to airframes owing to their higher flexibility and lightness compared to inorganic materials. Their properties originate from the chemical structures, chain conformations and entanglement structures of the polymer molecules. Thus, understanding the polymeric materials from the atomic and molecular level is essential to grasp the nature of polymers for further development of high-performance materials. Direct observation of molecular chain conformations and dynamics in polymeric materials is thus an effective way for this purpose.

Transmission electron microscopy (TEM) and scanning transmission electron microscopy (STEM) is the sole atomic-resolution observation tools for the internal structures of materials. However, because polymers are naturally low contrast and electron-beam sensitive materials for electrons, high-resolution TEM/STEM observation of polymer-chain conformations is still a challenging subject: such observations are limited to the chains along the edges of nanofibers [1].

The annular dark-field STEM (ADF-STEM) allows us to identify the positions of heavy atoms. ADFSTEM has been used to observe heavy atoms in organic materials having conductivity (ionic liquids) as well as inorganic ones [2,3]. In the present study, we have challenged the direct observation of the chain structures of individual polymers using ADF-STEM to a labeled polymer with heavy atoms and a conductive substrate.

As the labeled polymer, poly(4-iodostyrene), that is iodine-labeled polystyrene, was synthesized by anionic polymerization of 4-iodostyrene (molecular weight $M_{n}: 4,700$ ), whose molecular structure is shown in Fig. 1a. The atomic number of iodine $(Z=53)$ is much larger than those of the other constituent elements $(\mathrm{H}, \mathrm{C})$; thus, the iodine atoms are imaged much brighter with ADF-STEM (Fig. 1b). The conductive and flat support films were prepared by transferring the graphene sheets exfoliated from a highly oriented pyrolytic graphite onto QUANTIFOIL (Quantifoil Micro Tools GmbH, Germany). Subsequently, poly(4-iodostyrene) was cast with toluene (1 wt\% solution) and dispersed on the graphene sheets. The ADF-STEM observation was performed on this sample.

At the conference time, we will report our recent successful observation to identify partial conformations of individual polymer chains from the spot arrays of the iodine atoms. 
a<smiles>FC(c1ccc(I)cc1)C(F)(F)F</smiles>

b

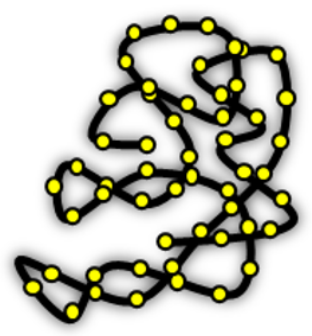

Figure 1. a, Molecular structure of poly(4-iodostyrene). b, Schematic of the polymer chain conformation. The iodine atoms are observed bright with ADF-STEM.

\section{Observation}

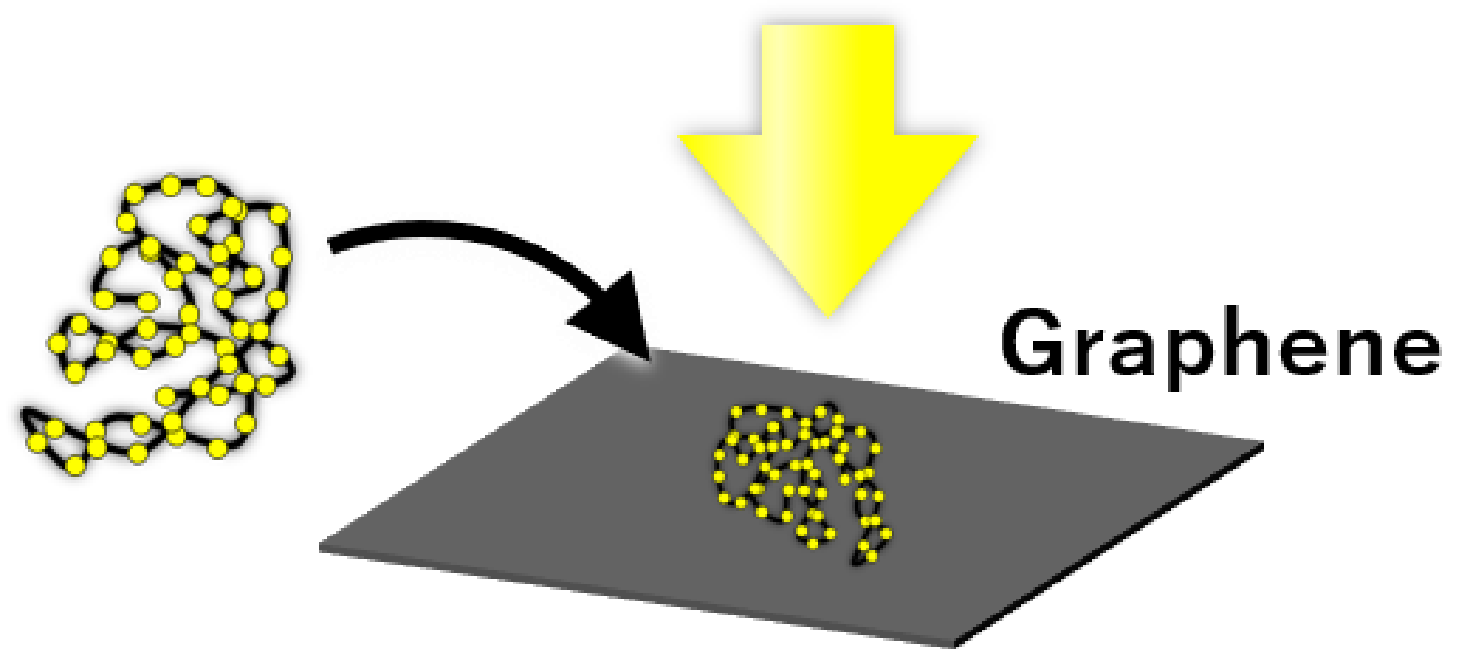

Figure 2. Observation schematic. Molecules of poly(4-iodostyrene) were put on graphene sheets.

\section{References}

[1] Z. Zhong et al., Polymer 54, 3745 (2013).

[2] T. Miyata, F. Uesugi and T. Mizoguchi, Science Advances 3, e1701546 (2017).

[3] T. Miyata and T. Mizoguchi, Microscopy 67, i162 (2018). 